\title{
Distortion Bounds for Broadcasting a Gaussian Source in the Presence of Interference
}

\author{
Ahmad Abou Saleh, Fady Alajaji, and Wai-Yip Chan \\ Queen's University, Kingston, ON, K7L 3N6 \\ Email: ahmad.abou.saleh@queensu.ca, fady@mast.queensu.ca, chan@queensu.ca
}

\begin{abstract}
We consider the transmission of a Gaussian source over the two-user Gaussian broadcast channel in the presence of interference that is known to the transmitter. The interference is assumed to be correlated to the source and each user is interested in estimating the source signal. We propose a hybrid digitalanalog (HDA) scheme based on proper combinations of power splitting, Wyner-Ziv and HDA Costa coding. The achievable (square-error) distortion region (inner bound) of this scheme is analyzed under source-channel bandwidth expansion; the matched bandwidth is treated as a special case. An outer bound on the distortion region is also derived by assuming full/partial knowledge of the interference at both users and by adapting the approach of Reznic et al. (2006). This outer bound can be tighter than the "trivial" outer bound found by assuming point-to-point communication.
\end{abstract}

\section{INTRODUCTION}

The traditional approach for analog source transmission over noisy channels is to use a tandem scheme that is composed of separate source and channel coders. This is well known to be optimal for point-to-point communications. For multi-terminal systems, tandem coding is no longer optimal. Joint sourcechannel coding (JSCC) may achieve better performance. One scenario where tandem coding is suboptimal concerns the broadcast of Gaussian sources over Gaussian channels [1].

For a single Gaussian source sent over a Gaussian broadcast channel with matched source-channel bandwidth, a linear scheme is optimal (e.g., see [1]). For mismatched sourcechannel bandwidth, the best known coding schemes uses hybrid signalling [2]-[5]. One extension to this problem is the broadcasting of two correlated sources to two users, each of which is interested in recovering one of the two sources; in [6], the authors focused on the matched sourcechannel bandwidth case and proved that the linear scheme is optimal when the system's signal-to-noise ratio is below a certain threshold. In [7], a hybrid digital-analog (HDA) scheme is proposed for the same scenario and is shown to be optimal whenever the linear scheme of [6] is not. Under mismatched bandwidth, several HDA schemes were proposed in [8], based on combinations of hybrid coding and dirty paper coding. Recently, in [9], a tandem scheme based on successive coding is studied and shown to outperform the HDA schemes of [8] for a range of system settings. In [10], the authors considered the transmission of a Gaussian source over a correlated interference channel and proposed a hybrid coding scheme for point-to-point communications. In [11], we

This work was supported in part by NSERC of Canada. investigate the transmission of bivariate Gaussian sources over a broadcast Gaussian channel with interference.

In this paper we consider a single source broadcast over a Gaussian channel with interference that is correlated to the source. In our previous work [11], we excluded the case when the bivariate sources are fully correlated (i.e., single source broadcast). This work, which focuses on this case, generalizes the results of [4] to the case of interference broadcast channels. This broadcast system with correlated source-interference can model situations where two nearby nodes are transmitting correlated information simultaneously. One node sends directly its signal; the other, however, has knowledge about its neighbour signal and treats it as correlated interference. We propose and analyze an HDA scheme for this system based on Wyner-Ziv [12], Costa [13] and HDA Costa coding [14] for source-channel bandwidth expansion (the matched case is treated as special case). The rest of the paper is organized as follows. Section II presents the problem formulation. Section III introduces outer bounds on the system's distortion region. In Section IV, an inner bound on the distortion region is derived. Numerical results are included in Section V and conclusions are drawn in Section VI.

\section{Problem Formulation}

We consider the transmission (Fig. 1) of a single memoryless Gaussian source $V$ over a two-user interference Gaussian broadcast channel. The interference $S$ is Gaussian and known to the transmitter. User $i(i=1,2)$ receives the transmitted signal disturbed by additive white Gaussian noise $W_{i}$ and interference $S$ with variances $\sigma_{W_{i}}^{2}$ and $\sigma_{S}^{2}$, respectively. Each user aims to estimate $V^{K}=(V(1), V(2), \ldots, V(K))$, where samples are drawn from an independent and identically distributed (i.i.d.) Gaussian distribution. In this paper, we assume that $(V(j), S(j)), j=1, \ldots, K$, are correlated via the following covariance matrix

$$
\Sigma_{V S}=\left[\begin{array}{cc}
\sigma_{V}^{2} & \rho \sigma_{V} \sigma_{S} \\
\rho \sigma_{V} \sigma_{S} & \sigma_{S}^{2}
\end{array}\right]
$$

where $\sigma_{V}^{2}$ is the variance of $V(j)$ and $\rho$ is the correlation coefficient between $V(j)$ and $S(j)$. As shown in Fig. 1, the source-interference pair vector $\left(V^{K}, S^{N}\right)$ is transformed into an $N$ dimensional channel input $X^{N} \in \mathbb{R}^{N}$ via $\alpha(\cdot)$, a mapping from $\left(\mathbb{R}^{K} \times \mathbb{R}^{N}\right) \rightarrow \mathbb{R}^{N}$. The received vector at user $i$ is $Y_{i}^{N}=X^{N}+S^{N}+W_{i}^{N}, i=1,2$, where addition is component-wise, $X^{N}=\alpha\left(V^{K}, S^{N}\right), S^{N}$ is the i.i.d. 


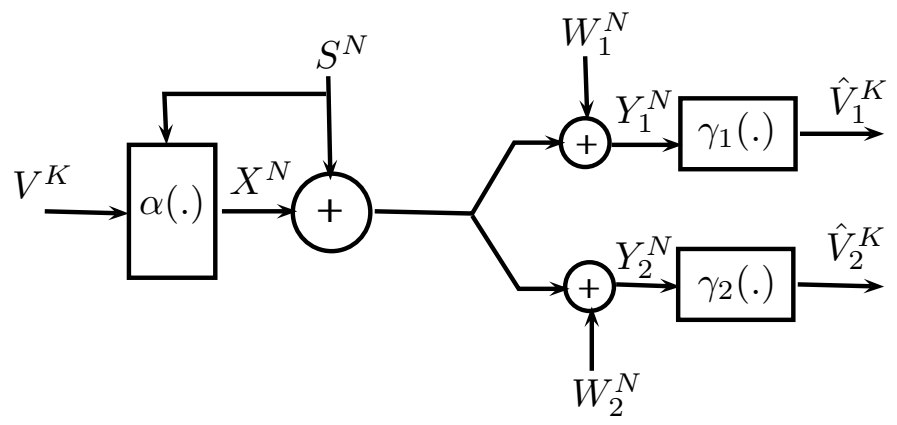

Fig. 1. System model.

Gaussian interference known to the transmitter, and samples in the additive noise $W_{i}^{N}$ are drawn from an i.i.d. Gaussian distribution $\left(\mathcal{N}\left(0, \sigma_{W_{i}}^{2}\right)\right)$ independently from both the source and the interference. The system operates under an average power constraint $P$ given by

$$
\mathbb{E}\left[\left\|\alpha\left(V^{K}, S^{N}\right)\right\|^{2}\right] / N \leq P
$$

where $\mathbb{E}[(\cdot)]$ denotes the expectation operator. The decoded signal at user $i$ is given by $\hat{V}_{i}^{K}=\gamma_{i}\left(Y_{i}^{N}\right) ; \gamma_{i}($.$) , which is the$ decoder function, is a mapping from $\mathbb{R}^{N} \rightarrow \mathbb{R}^{K}$. The system's rate is given by $\lambda=\frac{N}{K}$ channel use/source symbol and the reconstruction fidelity at each user is the mean square error (MSE) $D_{i}=\mathbb{E}\left[\left\|V^{K}-\hat{V}_{i}^{K}\right\|^{2}\right] / K$ for $i=1,2$. We assume that $\sigma_{W_{1}}^{2}>\sigma_{W_{2}}^{2}$ and that the broadcast channel is degraded. Thus, user 1 is considered the weak user and user 2 is the strong one. For a given power constraint $P$ and rate $\lambda$ the distortion region is defined as the closure of all distortion pairs $\left(\tilde{D}_{1}, \tilde{D}_{2}\right)$ for which $\left(P, \tilde{D}_{1}, \tilde{D}_{2}\right)$ is achievable, where a powerdistortion triple is achievable if for any $\delta>0$, there exist sufficiently large integers $K$ and $N$ with $N / K=\lambda$, encoding and decoding functions $\left(\alpha, \gamma_{1}, \gamma_{2}\right)$ satisfying (2), such that $D_{i}<\tilde{D}_{i}+\delta, i=1,2$. In this work, we are interested in analyzing the distortion region of this system under matched $(\lambda=1)$ and expansion bandwidth modes $(\lambda>1)$. Note that for $\lambda>1, V^{K}$ and the first $K$ interference samples $S^{K}$ in $S^{N}=\left[S^{K}, S^{N-K}\right]$ are correlated via the covariance matrix in (1), while $V^{K}$ and $S^{N-K}$ (the remaining $N-K$ samples in $S^{N}$ ) are independent.

\section{OUTER BOUNDS}

\section{A. Outer Bound 1}

In [10], [15], several bounds are derived for point-to-point communications under correlated interference and matched source-channel bandwidth. In this section, we derive an outer bound on the distortion region for the interference broadcast channel for $\lambda \geq 1$ by treating each user separately; we refer to this bound in some cases as the "trivial" bound. Since $S(j)$ and $V(j)$ are correlated for $j=1, \ldots, K$, we have $S(j)=S_{I}(j)+S_{D}(j)$, with $S_{D}(j)=\frac{\rho \sigma_{S}}{\sigma_{V}} V(j)$ and $S_{I}(j) \sim \mathcal{N}\left(0,\left(1-\rho^{2}\right) \sigma_{S}^{2}\right)$ are independent of each other. To derive an outer bound, we assume knowledge of $\left(\tilde{S}^{K}, S^{N-K}\right)$ at both users, where $\tilde{S}^{K}=\eta_{1} S_{I}^{K}+\eta_{2} S_{D}^{K}$ and $\left(\eta_{1}, \eta_{2}\right)$ is a pair of real parameters. The knowledge of the linear combination $\tilde{S}^{K}$ is motivated by [15]. Note that this outer bound gives a rectangular region.
Lemma 1. The outer bound on the distortion region under bandwidth expansion $(\lambda \geq 1)$ can be expressed as follows

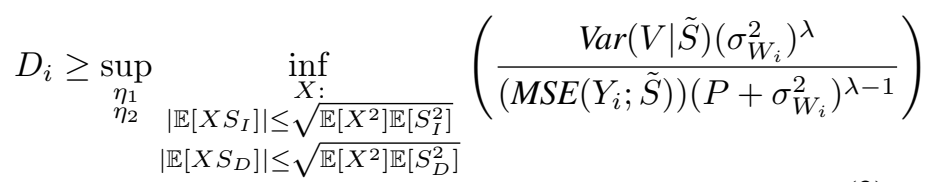

where $i=\{1,2\}, \operatorname{Var}(V \mid \tilde{S})=\sigma_{V}^{2}\left(1-\frac{\eta_{2}^{2} \rho^{2}}{\eta_{1}^{2}\left(1-\rho^{2}\right)+\eta_{2}^{2} \rho^{2}}\right)$ is the conditional variance of $V$ given $\tilde{S}$ and $\operatorname{MSE}\left(Y_{i} ; \tilde{S}\right)=$ $\mathbb{E}\left[Y_{i}^{2}\right]-\frac{\left(\mathbb{E}\left[Y_{i} \tilde{S}\right]\right)^{2}}{\mathbb{E}\left[\tilde{S}^{2}\right]}$ is the distortion from estimating $Y_{i}$ based on $\tilde{S}$ using a linear minimum MSE estimator (LMMSE).

Proof: For a $K: N$ system with $N \geq K$, we have

$$
\begin{aligned}
\frac{K}{2} \log \frac{\operatorname{Var}(V \mid \tilde{S})}{D_{i}} & \leq I\left(V^{K} ; \hat{V}_{i}^{K} \mid \tilde{S}^{K}, S^{N-K}\right) \\
& \leq I\left(V^{K} ; Y_{i}^{N} \mid \tilde{S}^{K}, S^{N-K}\right) \\
& =h\left(Y_{i}^{N} \mid \tilde{S}^{K}, S^{N-K}\right)-h\left(W_{i}^{N}\right)
\end{aligned}
$$

where (4) can be upper bounded as follows

$$
\begin{aligned}
& h\left(Y_{i}^{K} \mid \tilde{S}^{K}\right)+h\left(Y_{i}^{N-K} \mid S^{N-K}\right)-h\left(W^{N}\right) \\
\leq & \frac{K}{2} \log 2 \pi e\left(\operatorname{MSE}\left(Y_{i} ; \tilde{S}\right)\right)+\frac{N-K}{2} \log 2 \pi e\left(P+\sigma_{W_{i}}^{2}\right) \\
& -\frac{N}{2} \log 2 \pi e\left(\sigma_{W_{i}}^{2}\right)
\end{aligned}
$$

where we used $h\left(Y_{i}^{K} \mid \tilde{S}^{K}\right) \leq h\left(Y_{i}^{K}-\gamma_{\text {lmse }}\left(\tilde{S}^{K}\right)\right) \leq$ $\frac{K}{2} \log 2 \pi e\left(\mathbb{E}\left[\left(Y_{i}-\gamma_{\operatorname{lmse}}(\tilde{S})\right)^{2}\right]\right)=\frac{K}{2} \log 2 \pi e\left(\operatorname{MSE}\left(Y_{i} ; \tilde{S}\right)\right)$, where $\gamma_{\text {lmse }}\left(\tilde{S}^{K}\right)$ is the LMMSE estimator of $Y_{i}$ based on $\tilde{S}$. $\operatorname{MSE}\left(Y_{i} ; \tilde{S}\right)$ is a function of $\eta_{1}, \eta_{2}, \mathbb{E}\left[X S_{I}\right]$ and $\mathbb{E}\left[X S_{D}\right]$. By Cauchy-Schwarz, we have $\left|\mathbb{E}\left[X S_{I}\right]\right| \leq \sqrt{\mathbb{E}\left[X^{2}\right] \mathbb{E}\left[S_{I}^{2}\right]}$ and $\left|\mathbb{E}\left[X S_{D}\right]\right| \leq \sqrt{\mathbb{E}\left[X^{2}\right] \mathbb{E}\left[S_{D}^{2}\right]}$. For a given $\eta_{1}$ and $\eta_{2}$, the maximum value of $\operatorname{MSE}\left(Y_{i} ; S\right)$ has to be used in (5). Note that all inequalities follow from rate-distortion theory, the data processing inequality, the chain rules for mutual information and differential entropy, the property that conditioning reduces differential entropy and the fact that the Gaussian distribution maximizes differential entropy.

\section{B. Outer Bound 2}

To derive this bound, we need to introduce an auxiliary random variable $U^{K}$ similar to the one in [16]. This approach was also used to find a bound for the Gaussian broadcast channel [4]. An outer bound can be obtained by assuming knowledge of $\left(\tilde{S}^{K}, S^{N-K}\right)$ at both users and choosing $U^{K}=$ $V^{K}+Z^{K}$, where $Z^{K}$ is independent of everything else and samples of $Z^{K}$ follow a zero mean i.i.d. Gaussian distribution with variance $\sigma_{Z}^{2}=\xi \sigma_{V}^{2}$ and $\xi>0$.

Lemma 2. For a $K: N$ bandwidth expansion system, the outer bound can be expressed as follows

$$
\begin{aligned}
& D_{1}=\eta \frac{\operatorname{Var}(V \mid \tilde{S})\left(\sigma_{W_{1}}^{2}\right)^{\lambda}}{\left(\operatorname{MSE}\left(Y_{1} ; \tilde{S}\right)\right)\left(P+\sigma_{W_{1}}^{2}\right)^{\lambda-1}} \\
& D_{2} \geq \sup _{\xi} \frac{\operatorname{Var}(V \mid \tilde{S}) \sigma_{Z}^{2}\left(\sigma_{W_{2}}^{2}\right)^{\lambda}}{\operatorname{Var}(U \mid \tilde{S})\left[A\left(\frac{D_{1}+\sigma_{Z}^{2}}{\operatorname{Var}(U \mid \tilde{S})}\right)^{\frac{1}{\lambda}}-\sigma_{\tilde{W}}^{2}\right]^{\lambda}-B}
\end{aligned}
$$


where $\eta \geq 1, A=\left(\operatorname{MSE}\left(Y_{1} ; \tilde{S}\right)\right)^{\frac{1}{\lambda}}\left(P+\sigma_{W_{1}}^{2}\right)^{\frac{\lambda-1}{\lambda}}, B=$ $\left(\sigma_{W_{2}}^{2}\right)^{\lambda} \operatorname{Var}(V \mid \tilde{S}), \quad \sigma_{\tilde{W}}^{2}=\sigma_{W_{1}}^{2}-\sigma_{W_{2}}^{2}$ and $\operatorname{Var}(U \mid \tilde{S})=$ $\operatorname{Var}(V \mid \tilde{S})+\sigma_{Z}^{2}$ is the variance of $U$ given $\tilde{S}$. Note that the outer bound has to be maximized over $\eta_{1}$ and $\eta_{2}$ and that the bound on $D_{2}$ is a function of $D_{1}$.

Proof: $D_{1}$ has the same form as the one in Lemma 1; for a given $\eta \geq 1$, we get a bound on $D_{2}$. This is done as follows

$$
\begin{aligned}
& \frac{K}{2} \log \frac{\operatorname{Var}(V \mid \tilde{S})}{D_{2}} \leq I\left(V^{K} ; \hat{V}_{2}^{K} \mid \tilde{S}^{K}, S^{N-K}\right) \\
\leq \quad & I\left(V^{K} ; Y_{2}^{N} \mid \tilde{S}^{K}, S^{N-K}\right) \\
= & I\left(V^{K} ; U^{K} \mid \tilde{S}^{K}, S^{N-K}\right)+I\left(V^{K} ; Y_{2}^{N} \mid U^{K}, \tilde{S}^{K}, S^{N-K}\right) \\
& -I\left(V^{K} ; U^{K} \mid Y_{2}^{N}, \tilde{S}^{K}, S^{N-K}\right) \\
\leq \quad & h\left(U^{K} \mid \tilde{S}^{K}, S^{N-K}\right)-h\left(U^{K} \mid Y_{2}^{N}, \tilde{S}^{K}, S^{N-K}\right) \\
& +h\left(Y_{2}^{N} \mid U^{K}, \tilde{S}^{K}, S^{N-K}\right)-h\left(W_{2}^{N}\right) .
\end{aligned}
$$

Note that $h\left(U^{K} \mid \tilde{S}^{K}, S^{N-K}\right)=\frac{K}{2} \log 2 \pi e \operatorname{Var}(U \mid \tilde{S})$ and $h\left(W_{2}^{N}\right)=\frac{N}{2} \log 2 \pi e \sigma_{W_{2}}^{2}$. Next, we bound the remaining two terms in (7). Using the entropy power inequality, we have $2^{\frac{2}{K} h\left(U^{K} \mid Y_{2}^{N}, \tilde{S}^{K}, S^{N-K}\right)} \geq 2^{\frac{2}{K} h\left(V^{K} \mid Y_{2}^{N}, \tilde{S}^{K}, S^{N-K}\right)}+2^{\frac{2}{K} h\left(Z^{K}\right)}$.

Now using (8) and the fact that $h\left(V^{K} \mid Y_{2}^{N}, \tilde{S}^{K}, S^{N-K}\right)=$ $h\left(V^{K} \mid \tilde{S}^{K}, S^{N-K}\right)-I\left(V^{K} ; Y_{2}^{N} \mid \tilde{S}^{K}, S^{N-K}\right)$, we can lower bound $h\left(U^{K} \mid Y_{2}^{N}, \tilde{S}^{K}, S^{N-K}\right)$ by

$$
\frac{K}{2} \log 2 \pi e\left(\operatorname{Var}(V \mid \tilde{S}) 2^{-\frac{2}{K} I\left(V^{K} ; Y_{2}^{N} \mid \tilde{S}^{K}, S^{N-K}\right)}+\sigma_{Z}^{2}\right)
$$

Since the broadcast channel is degraded, $Y_{1}^{N}$ can be written as the sum of $Y_{2}^{N}$ and a noise $\tilde{W}$ that is independent of everything else and has a variance $\sigma_{\tilde{W}}^{2}=\sigma_{W_{1}}^{2}-\sigma_{W_{2}}^{2}$. Hence using the entropy power inequality, we have

$$
2^{\frac{2}{N} h\left(Y_{1}^{N} \mid U^{K}, \tilde{S}^{K}, S^{N-K}\right)} \geq 2^{\frac{2}{N} h\left(Y_{2}^{N} \mid U^{K}, \tilde{S}^{K}, S^{N-K}\right)}+2 \pi e \sigma_{\tilde{W}}^{2}
$$

where $h\left(Y_{1}^{N} \mid U^{K}, \tilde{S}^{K}, S^{N-K}\right)=h\left(Y_{1}^{N} \mid \tilde{S}^{K}, S^{N-K}\right)-$ $I\left(Y_{1}^{N} ; U^{K} \mid \tilde{S}^{K}, S^{N-K}\right) ; h\left(Y_{1}^{N} \mid \tilde{S}^{K}, S^{N-K}\right)$ can be upper bounded in a similar way as in (5) and $I\left(Y_{1}^{N} ; U^{K} \mid \tilde{S}^{K}, S^{N-K}\right)$ is lower bounded as follows

$$
\begin{aligned}
I\left(Y_{1}^{N} ; U^{K} \mid \tilde{S}^{K}, S^{N-K}\right) & \geq I\left(\hat{V}_{1}^{K} ; U^{K} \mid \tilde{S}^{K}, S^{N-K}\right) \\
& \geq \frac{K}{2} \log \frac{\operatorname{Var}(U \mid \tilde{S})}{D_{1}+\sigma_{Z}^{2}}
\end{aligned}
$$

where we used the data processing theorem, the rate-distortion theory and the fact that $\frac{1}{K} \mathbb{E}\left[\left\|\hat{V}_{1}^{K}-U^{K}\right\|^{2}\right]=\left(D_{1}+\sigma_{Z}^{2}\right)$ [4]. Now combining all the above inequalities and after some manipulations we can get the bound on $D_{2}$ given in (6).

\section{HDA CODING SCHEME}

In this section, we present a layered scheme for the broadcast channel in the presence of interference. This scheme comprises two layers that are concatenated to output the transmitted signal as shown in Fig. 2. The first layer has three sublayers that are merged to output $X_{1}^{K}$. The first sublayer, which uses an average power of $P_{a}$, outputs $X_{a}^{K}=\sqrt{a}\left(\beta_{1} V^{K}+\beta_{2} S^{K}\right)$, a linear combination of the source and the interference, where $\beta_{1}, \beta_{2} \in[-1,1]$, and $a=P_{a} /\left(\beta_{1}^{2} \sigma_{V}^{2}+\beta_{2}^{2} \sigma_{S}^{2}+2 \beta_{1} \beta_{2} \rho \sigma_{V} \sigma_{S}\right)$ is a gain factor related to power constraint $P_{a}$; this sublayer is used to benefit from the correlation between the source and the interference. The second sublayer, which outputs $X_{11}^{K}$ with power $P_{11}$, uses HDA Costa coding on the source $V^{K}$. This layer is meant for both users and treats $X_{a}^{K}$ and $S^{K}$ as known interference. The auxiliary random variable of the HDA Costa encoder is $U_{11}^{K}=X_{11}^{K}+\alpha_{11}\left(S^{K}+X_{a}^{K}\right)+$ $\kappa_{11} V^{K}$, where $X_{11} \sim \mathcal{N}\left(0, P_{11}\right), \alpha_{11}=\frac{P_{11}}{P-P_{a}+\sigma_{W_{1}}^{2}}, \kappa_{11}^{2}=$ $\frac{P_{11}^{2}}{\left(P-P_{a}+\sigma_{W_{1}}^{2}\right) D_{1 a}}$, and $D_{1 a}$ is defined in (12) below. The HDA Costa encoder forms a codebook $\mathcal{U}_{11}$ with codeword length $K$ and $2^{K R_{11}}$ codewords ( $R_{11}$ is defined later). Every codeword is generated following the random variable $U_{11}^{K}$. The codebook is revealed to both the encoder and decoder. The encoder searches for a $U_{11}^{K} \in \mathcal{U}_{11}$ such that $\left(V^{K},\left(S^{K}+X_{a}^{K}\right), U_{11}^{K}\right)$ are jointly typical. The third sublayer, which is meant for the strong user, encodes the source $V^{K}$ using Wyner-Ziv coding at a rate $R_{12}=\frac{1}{2} \log \left(1+\frac{P_{12}}{P-P_{a}-P_{11}+\sigma_{W_{2}}^{2}}\right)$. The Wyner-Ziv index is then encoded using Costa coding that treats $S^{K}$, $X_{a}^{K}$, and $X_{11}^{K}$ as interference and uses the remaining power $P_{12}=P-P_{a}-P_{11}$; the output of this sublayer is $X_{12}^{K}$.

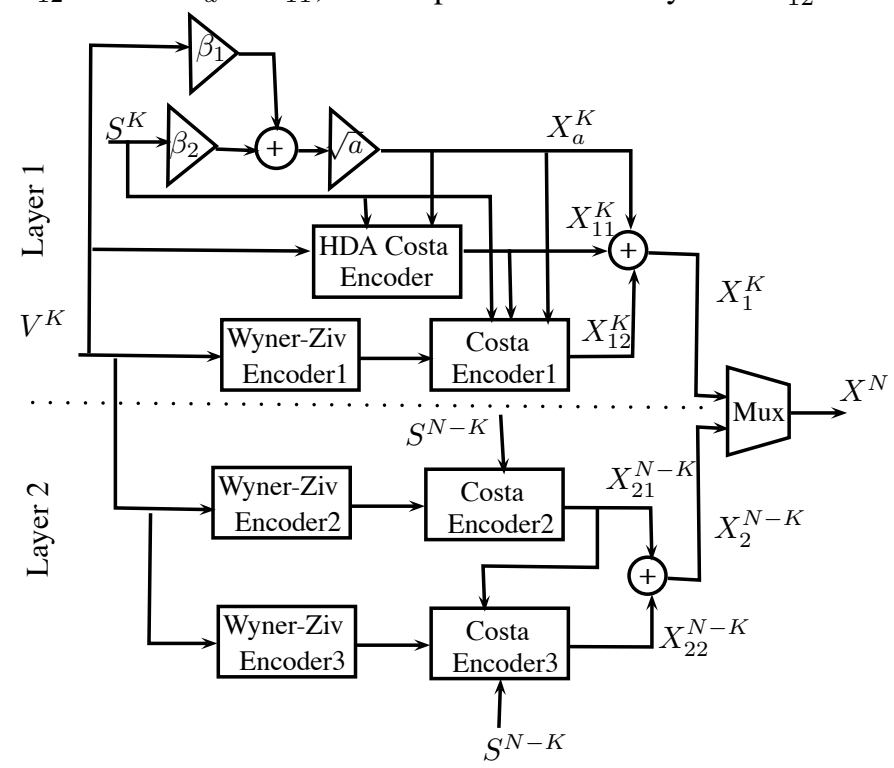

Fig. 2. HDA scheme encoder structure for rate $\lambda=\frac{N}{K} \geq 1$.

The second layer, which outputs $X_{2}^{N-K}$, is composed of two sublayers. The first sublayer encodes $V^{K}$ using a WynerZiv at a rate $R_{21}=\frac{1}{2} \log \left(1+\frac{P_{21}}{P-P_{21}+\sigma_{W_{1}}^{2}}\right)$ followed by a Costa coder with an average power $P_{21}$. Note that the Costa coder treats $S^{N-K}$ as interference and outputs $X_{21}^{N-K}$ that is meant for both users. The second sublayer encodes $V^{K}$ using a Wyner-Ziv at a rate $R_{22}=\frac{1}{2} \log \left(1+\frac{P_{22}}{\sigma_{W_{2}}^{2}}\right)$ followed by a Costa coder with an average power $P_{22} \stackrel{W_{2}}{=} P-P_{21}$ that treats $X_{21}^{N-K}$ and $S^{N-K}$ as interference and outputs $X_{22}^{N-K}$. The output of the second layer is then given by $X_{2}^{N-K}=$ $X_{21}^{N-K}+X_{22}^{N-K}$. Note that $X^{N}$ is the concatenation of $X_{1}^{K}$ and $X_{2}^{N-K}$.

As shown in Fig. 3, at the weak user, from the first $K$ samples of the noisy received signal $Y_{1}^{N}=\left[Y_{1}^{K} Y_{1}^{N-K}\right]$, a LMMSE estimator is used to get an estimate of $V^{K}$ denoted by $\hat{V}_{1 a}^{K}$ based on $Y_{1}^{K}$. The distortion $D_{1 a}=\mathbb{E}\left[\left\|V^{K}-\hat{V}_{1 a}^{K}\right\|^{2}\right] / K$, 
which is used in the parameter $\kappa_{11}$, is given by

$$
D_{1 a}=\sigma_{V}^{2}-\frac{\mathbb{E}\left[V Y_{1}\right]^{2}}{\mathbb{E}\left[Y_{1}^{2}\right]}=\sigma_{V}^{2}-\frac{\left(\mathbb{E}\left[V X_{a}\right]+\mathbb{E}[V S]\right)^{2}}{P+\sigma_{S}^{2}+\sigma_{W_{1}}^{2}+2 \mathbb{E}\left[S X_{a}\right]} .
$$

With our choice of parameters $\alpha_{11}$ and $\kappa_{11}$, the HDA Costa decoder can estimate $U_{11}^{K}$ with low probability of error (for $K$ sufficiently large) by searching for a $U_{11}^{K}$ such that $\left(U_{11}^{K}, Y_{1}^{K}, \hat{V}_{1 a}^{K}\right)$ are jointly typical. Note that the HDA codeword $U_{11}^{K}$ can be decoded at both users. This can be proved by noting that the HDA Costa rate $R_{11}$ satisfies $I\left(U_{11} ;\left(S+X_{a}\right), V\right) \leq R_{11} \leq I\left(U_{11} ; Y_{i}, \hat{V}_{i a}\right)$, for $i=1,2$, where $\hat{V}_{2 a}$ is the LMMSE estimate of $V$ based on the received signal at the strong user. The HDA Costa decoder then forms a LMMSE estimate of $V^{K}$, denoted by $\hat{V}_{1}^{\prime K}$, based on $Y_{1}^{K}$ and the decoded codeword $U_{11}^{K}$. The resulting distortion is

$$
D_{1}^{\prime}=\sigma_{V}^{2}-\Gamma_{1}^{T} \Lambda_{1}^{-1} \Gamma_{1}
$$

where $\Lambda_{1}$ is the covariance matrix of $\left[\begin{array}{ll}U_{11} & Y_{1}\end{array}\right]$, and $\Gamma_{1}$ is the correlation vector between $V$ and $\left[\begin{array}{ll}U_{11} & Y_{1}\end{array}\right]$. Note that after some manipulations, the distortion in (13) can be written as

$$
D_{1}^{\prime}=\frac{D_{1 a}}{1+P_{11} /\left(P-P_{a}-P_{11}+\sigma_{W_{1}}^{2}\right)} .
$$

A better estimate of $V^{K}$ at the weak user is obtained by using the decoded Wyner-Ziv 2 codeword $T_{2}^{K}$ and the previous estimate $\hat{V}_{1}^{\prime} K$. Note that $T_{2}^{K}=\alpha_{w z 2} V^{K}+H_{2}$, where $\alpha_{w z 2}=\sqrt{1-\left(\frac{P_{22}+\sigma_{W_{1}}^{2}}{P_{21}+P_{22}+\sigma_{W_{1}}^{2}}\right)^{\lambda-1}}$, and $H_{2} \sim$ $\mathcal{N}\left(0, D_{1}^{\prime} /\left(1+\frac{P_{21}}{P_{22}+\sigma_{W_{1}}^{2}}\right)^{\lambda-1}\right)$. The overall distortion in reproducing $V^{K}$ is then given by

$$
D_{1}=\frac{D_{1}^{\prime}}{\left(1+\frac{P_{21}}{P_{22}+\sigma_{W_{1}}^{2}}\right)^{\lambda-1}} .
$$

The above distortion can be achieved using a LMMSE estimator of $V^{K}$ based on $Y_{1}^{K}, U_{11}^{K}$ and $T_{2}^{K}$.

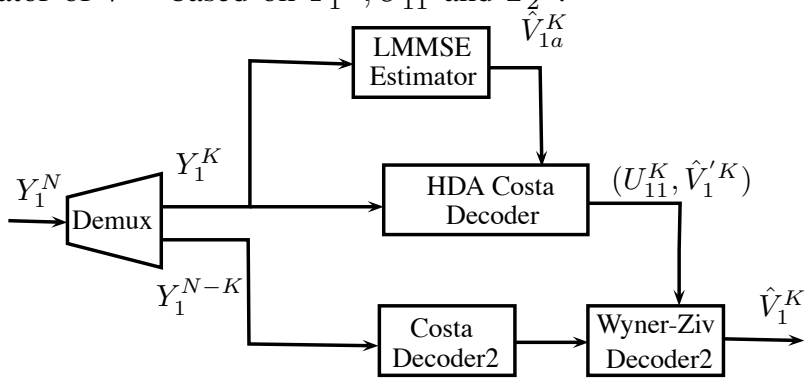

Fig. 3. HDA scheme decoder structure for the weak user.

The strong user, that is able to decode all codewords used by the weak user, estimates the source $V^{K}$ by first finding a linear MMSE estimate of $V^{K}$, denoted by $\hat{V}_{2}^{\prime} K$, based on the HDA Costa codeword $U_{11}^{K}$, the Wyner-Ziv 2 codeword $T_{2}^{K}$, and the first $K$ samples of the received noisy signal at the strong user $Y_{2}^{K}$. The distortion in reproducing $V^{K}$ at the strong user is then given by

$$
D_{2}^{\prime}=\sigma_{V}^{2}-\Gamma_{2}^{T} \Lambda_{2}^{-1} \Gamma_{2}
$$

where $\Lambda_{2}$ is the covariance matrix of $\left[\begin{array}{lll}U_{11} & Y_{2} & T_{2}\end{array}\right]$, and $\Gamma_{2}$ is the correlation vector between $V$ and $\left[\begin{array}{lll}U_{11} & Y_{2} & T_{2}\end{array}\right]$. A better refinement $\hat{V}_{2}^{K}$ is then found using the decoded Wyner-Ziv 1 codeword $T_{1}^{K}$ and the decoded Wyner-Ziv 3 codeword $T_{3}^{K}$. The resulting distortion in estimating $V^{K}$ at the strong user is

$$
D_{2}=\frac{D_{2}^{\prime}}{\left(1+\frac{P_{12}}{\sigma_{W_{2}}^{2}}\right)\left(1+\frac{P_{22}}{\sigma_{W_{2}}^{2}}\right)^{\lambda-1}}
$$

The above distortion is achieved using a LMMSE based on $U_{11}, Y_{2}, T_{1}, T_{2}$ and $T_{3}$. Note that $T_{1}^{K}=\alpha_{w z 1} V^{K}+$ $H_{1}$ and $T_{3}^{K}=\alpha_{w z 3} V^{K}+H_{3}$, where $\alpha_{w z 1}=$ $\sqrt{1-\left(1+P_{12} / \sigma_{W_{2}}^{2}\right)^{-1}}, H_{1} \sim \mathcal{N}\left(0, D_{2}^{\prime} /\left(1+\frac{P_{12}}{\sigma_{W_{2}}^{2}}\right)\right), \alpha_{w z 3}=$ $\sqrt{1-\left(1+P_{22} / \sigma_{W_{2}}^{2}\right)^{1-\lambda}}$ and $H_{3} \sim \mathcal{N}\left(0, D_{2}\right)$. The inner bound for the proposed HDA scheme is then given by (15) and (17). Note that for the special case of matched source-channel bandwidth, the layer that outputs $X_{2}^{N-K}$ is shut down. The inner bound can be found by setting $\lambda$ to 1 in (15) and (17), with $\Lambda_{2}$ is the covariance matrix of $\left[\begin{array}{ll}U_{11} & Y_{2}\end{array}\right]$, and $\Gamma_{2}$ is the correlation vector between $V$ and $\left[\begin{array}{ll}U_{11} & Y_{2}\end{array}\right]$.

Remark. For full correlation between the source and the interference $(\rho=1)$, an uncoded scheme, which is a scaled version of the source, is optimal for the matched bandwidth case (i.e., $\lambda=1$ ). This can be proved by comparing the resulting distortion to outer bound 1 . Note that the best outer bound for $\rho=1$ is obtained by choosing $\eta_{1}=1$ and $\eta_{2}=0$ in (3). This result is analogous to the one in [17] for pointto-point communication.

\section{NumERICAL RESUltS}

In this section, we assume that the source has variance $\sigma_{V}^{2}=$ 1 and is broadcasted to two users with interference variance $\sigma_{S}^{2}=1 \mathrm{~dB}$, and observation noise variance $\sigma_{W_{1}}^{2}=0 \mathrm{~dB}$ and $\sigma_{W_{2}}^{2}=-5 \mathrm{~dB}$, respectively. The system's average power is set to $P=1$. To evaluate the performance, we plot the inner and outer bounds derived in the previous sections.

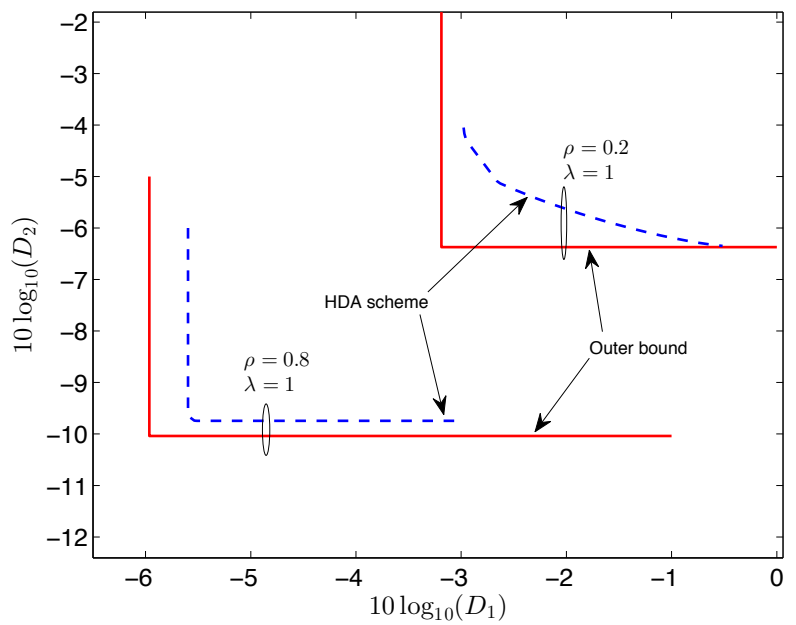

Fig. 4. Distortion region for the HDA scheme for $\lambda=1$.

Fig. 4 focuses on the matched source-channel bandwidth case $(\lambda=1)$. As we can notice, the gap between the inner 
and the outer bound decreases with an increase in the sourceinterference correlation $\rho$. We also noticed that the power allocated to the analog part of our scheme increases with $\rho$. For the extreme case of full correlation between the source and the interference $(\rho=1)$, our scheme reduces to a purely analog scheme which is optimal as mentioned in the remark. For the matched case, the best outer bound is found to be outer bound 1 (the trivial bound); using an auxiliary random variable as done in outer bound 2 is not useful in this case.

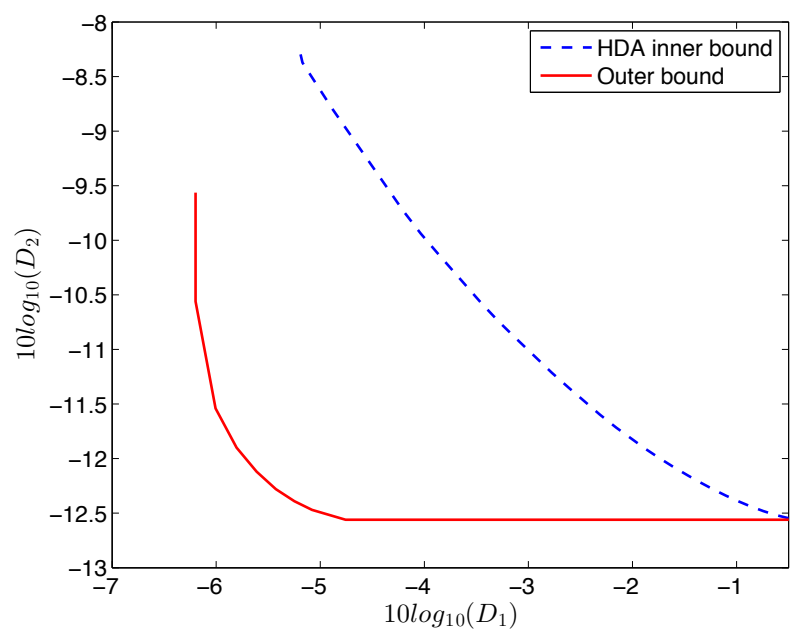

Fig. 5. Distortion region for the HDA scheme for $\lambda=2$ and $\rho=0.2$.

Figs. 5 and 6 show the inner and outer bounds for $\lambda=2$. We can notice that the gap between the achievable region and the outer bound is bigger than the ones in the matched bandwidth case. Moreover, outer bound 2 is shown to be beneficial in the expansion case. We can also notice that the proposed scheme performs close to the outer bound when $D_{1}$ is high; this is because for high distortion $D_{1}$, the system behaves similar to a point-to-point communication. Note that for the special case of $\lambda=2$ (our scheme is designed for any $\lambda \geq 1$ ), using HDA Costa coder at the expansion layer leads to an increase in the achievable distortion region.

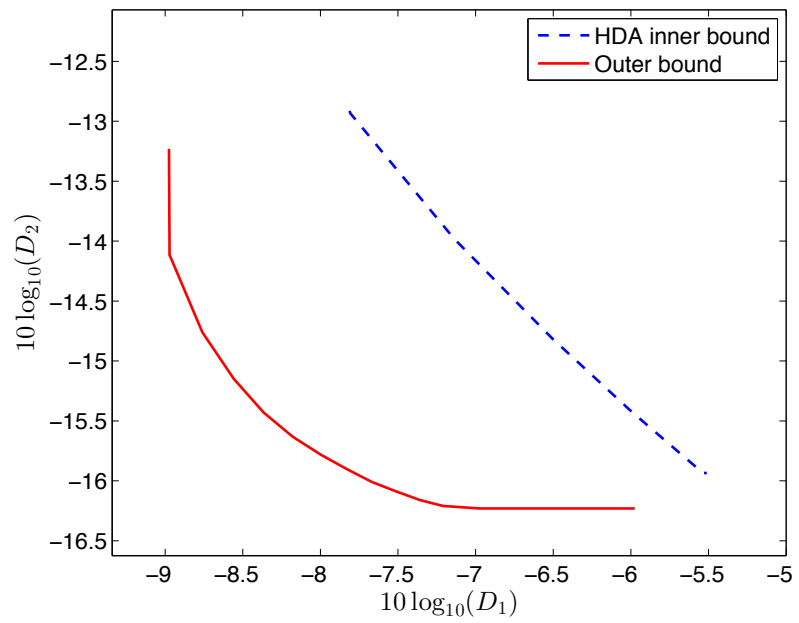

Fig. 6. Distortion region for the HDA scheme for $\lambda=2$ and $\rho=0.8$.

\section{SUMmARY AND CONCLUSIONS}

In this paper, we consider the transmission of a Gaussian source over the two-user degraded Gaussian broadcast channel in the presence of interference that is correlated to the source. We propose a layered HDA scheme for matched and expansion bandwidth scenarios based on Wyner-Ziv and HDA Costa coding; an inner bound (achievable distortion region) is derived for the HDA scheme. Outer bounds on the system's distortion region are also established by assuming additional knowledge at the receiver side and following the approach in [4]. For the fully correlated source-interference case under matched source-channel bandwidth, our HDA scheme, which reduces to a purely analog scheme, is shown to be optimal. Numerical results indicate that the HDA scheme performs close to the derived outer bounds under some system settings.

\section{REFERENCES}

[1] M. Gastpar, B. Rimoldi, and M. Vetterli, "To code, or not to code: lossy source-channel communication revisited," IEEE Trans. Inform. Theory, vol. 49, no. 5, pp. 1147-1158, May 2003

[2] U. Mittal and N. Phamdo, "Hybrid digital analog (HDA) joint source channel codes for broadcasting and robust communications," IEEE Trans. Inform. Theory, vol. 48, pp. 1082-1102, May 2002.

[3] M. Skoglund, N. Phamdo, and F. Alajaji, "Hybrid digital-analog sourcechannel coding for bandwidth compression/expansion," IEEE Trans. Inform. Theory, vol. 52, no. 8, pp. 3757-3763, Aug 2006.

[4] Z. Reznic, M. Feder, and R. Zamir, "Distortion bounds for broadcasting with bandwidth expansion," IEEE Trans. Inform. Theory, vol. 52, no. 8, pp. 3778-3788, Aug 2006.

[5] V. M. Prabhakaran, R. Puri, and K. Ramachandran, "Hybrid analogdigital strategies for source-channel broadcast," in Proc. 43rd Allerton Conf. Communication, Control and Computing, Allerton, IL, Sep 2005.

[6] A. Lapidoth and S. Tinguely, "Broadcasting correlated Gaussians," IEEE Trans. Inform. Theory, vol. 56, no. 7, pp. 3057-3068, July 2010.

[7] C. Tian, S. N. Diggavi, and S. Shamai, "The achievable distortion region of sending a bivariate Gaussian source on the Gaussian broadcast channel," IEEE Trans. Inform. Theory, vol. 57, no. 10, pp. 6419-6427, Oct 2011.

[8] H. Behroozi, F. Alajaji, and T. Linder, "On the performance of hybrid digital-analog coding for broadcasting correlated Gaussian sources," IEEE Trans. Communications, vol. 59, no. 12, pp. 3335-3342, Dec 2011.

[9] Y. Gao and E. Tuncel, "Separate source-channel coding for transmitting correlated Gaussian sources over degraded broadcast channels," IEEE Trans. Inform. Theory, vol. 59, no. 6, pp. 3619-3634, June 2013.

[10] Y.-C. Huang and K. R. Narayanan, "Joint source-channel coding with correlated interference," IEEE Trans. Commun., vol. 60, no. 5, pp. 13151327, May 2012.

[11] A. Abou Saleh, F. Alajaji, and W.-Y. Chan, "Hybrid digital-analog coding for interference broadcast channels," in Proc. IEEE Int. Symp. on Inform. Theory, Istanbul, Turkey, July 2013.

[12] A. D. Wyner and J. Ziv, "The rate-distortion function for source coding with side information at the decoder," IEEE Trans. Inform. Theory, vol. 22, pp. 1-10, Jan 1976.

[13] M. Costa, "Writing on dirty paper," IEEE Trans. Inform. Theory, vol. 29, no. 3, pp. 439-441, May 1983.

[14] M. P. Wilson, K. R. Narayanan, and G. Caire, "Joint source-channel coding with side information using hybrid digital analog codes," IEEE Trans. Inform. Theory, vol. 56, no. 10, pp. 4922-4940, Oct 2010.

[15] Y.-K. Chia, R. Soundararajan, and T. Weissman, "Estimation with a helper who knows the interference," in Proc. IEEE Int. Symp. on Inform. Theory, Cambridge, MA, July 2012.

[16] L. Ozarow, "On a source-coding problem with two channels and three receivers," Bell Syst. Tech. J., vol. 59, no. 10, pp. 1909-1921, Dec 1980.

[17] A. Sutivong, M. Chiang, T. Cover, and Y.-H. Kim, "Channel capacity and state estimation for state-dependent Gaussian channels," IEEE Trans. Inform. Theory, vol. 51, no. 4, pp. 1486-1495, Apr 2005. 